\title{
Carpectomía proximal en paciente con artrosis de muñeca
}

\author{
Proximal row carpectomy in patient with wrist osteoarthritis
}

\author{
Díaz-López JJ,* Vázquez-Alonso MF, † Tovar-Beltrán O§
}

Instituto Mexicano del Seguro Social. Unidad Médica de Alta Especialidad en Traumatología y Ortopedia Lomas Verdes.

RESUMEN. La artrosis de la muñeca es un proceso degenerativo, postraumático o idiopático que provoca al paciente dolor, pérdida de la movilidad, inflamación y deformidad. Las opciones quirúrgicas incluyen: artrodesis total de muñeca que produce una mejoría del dolor y disminución de la inflamación, otros tratamientos que permiten movilidad relativa son las artrodesis parciales. Otra solución quirúrgica es la carpectomía o la resección de la primera hilera del carpo, de tal manera que constituya una nueva articulación entre el radio y la segunda fila del carpo, obteniendo una congruencia articular adecuada. Material y métodos: Estudio observacional, descriptivo, serie de casos. Se valoraron 15 pacientes con carpectomía proximal durante el período de Enero de 2007 a Agosto de 2009, a quienes se realizó medición de arcos de movilidad y fuerza mediante las escalas de Mayo-DASH. Resultados: En 80\% de los pacientes entre 35 y 64 años se encontró predominio del sexo masculino en $67 \%$. La mejoría del dolor fue evidente, pasando de una media 7.7 en el preoperatorio a 2.7 en el postoperatorio, $10 \%$ de los casos presentaron dolor residual. Conclusiones: La carpectomía proximal representa una alternativa terapéutica que permite conservar la movilidad con mejora del dolor en la artrosis de muñeca.

Palabras clave: Carpectomía proximal, artrosis, tratamiento.

\section{Nivel de evidencia: IV}

* Médico adscrito al Servicio de Cirugía de Mano.

‡ Médico Jefe de Servicio de Cirugía de Mano.

${ }^{\S}$ Médico Jefe de Servicio de Urgencias.

Dirección para correspondencia:

Dr. José Joaquín Díaz López

Av. Lomas Verdes Núm. 52, Col. Santa Cruz Acatlán,

CP. 53121, Naucalpan de Juárez, Tel. 53710800, ext. 28685

E-mail:dr.diaz@yahoo.com.mx

Este artículo puede ser consultado en versión completa en: www.medigraphic.com/actaortopedica
ABSTRACT. The arthrosis of the wrist is a degenerative, traumatic or idiopathic process, which cause problem for patient characterized by pain, loss of mobility, swelling and deformity of the affected wrist. A surgical alternative is proximal row carpectomy, which consists of resection of the lunate, scaphoid and triquetrum, forming a new joint between the radius and the distal row carpus. Material and methods: Observational, descriptive, case series. 15 patients were evaluated who underwent proximal row carpectomy during the period January 2007-August 2009, with clinical follow-up until august 2010 through mayo and DASH scores, measuring strength. Range of motion and pain. $80 \%$ of patients were between 35 and 64 years. The predominant sex was male in $67 \%$. The result was satisfactory in $73 \%$, according to the scale of Mayo. The DASH scale in the postoperative period also improves. Conclusions: The proximal carpectomy is a surgical alternative, it preserves some joint mobility, reduced pain and improved disability of the limb.

Keywords: Proximal carpectomy, arthrosis, treatment.

\section{Introducción}

La artrosis de muñeca es un proceso degenerativo, postraumático o idiopático que provoca un problema crónico para el paciente, se caracteriza por dolor, pérdida de movilidad, inflamación y deformidad de la muñeca afectada (Figura 1). Entre las enfermedades que pueden evolucionar a artrodesis de muñeca se incluye la seudoartrosis del escafoides, la osteonecrosis del escafoides no tratada, la enfermedad de Kienböck, la inestabilidad crónica del carpo o las secuelas de la fractura distal de radio. $^{1,2}$

Las secuelas de las fracturas y luxaciones del radio distal y del carpo son la causa más frecuente de artrosis postraumática a nivel de la muñeca. Otras causas fre- 

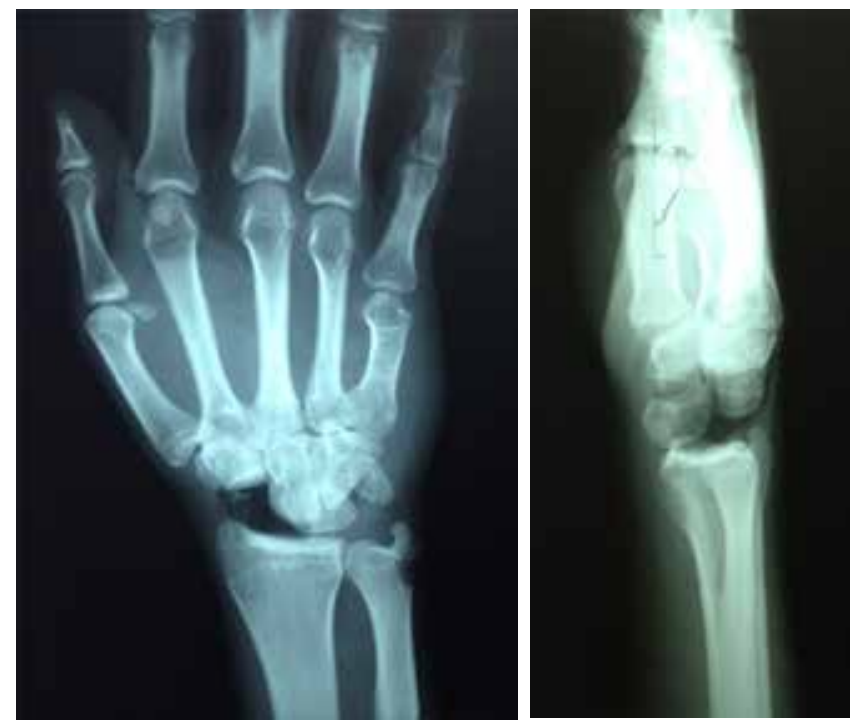

Figura 1: Luxación crónica del carpo caso 1.

cuentes son la seudoartrosis y la inestabilidad en flexión del escafoides, conocidas por las siglas SNAC (Scaphoid Nonunion Advanced Collapse) y SLAC (Scaphoid-Lunate Advance Collapse). El colapso avanzado escafolunar (SLAC), descrito por Watson en 1984, es la forma más común de artrosis degenerativa de la muñeca (55\%), ${ }^{2,3}$ además de la artrosis escafotrapecio trapezoide (26\%), una combinación de ambos patrones (14\%) y otras causas. ${ }^{4}$ El SLAC y el SNAC se dividen en tres tipos de acuerdo al grado de afección articular del carpo. En tipo I los cambios degenerativos comienzan entre la estiloides radial y el polo distal del escafoides tanto en la seudoartrosis del escafoides como en la disociación de la escafosemilunar. En el tipo II de la disociación escafosemilunar los cambios degenerativos progresan entre el radio distal y el polo proximal del escafoides. En cambio en la seudoartrosis de escafoides este espacio permanece respetado y la afección progresa hacia la articulación escafo-hueso grande. En el tipo III de ambas entidades se afecta la articulación hueso grande-semilunar respetando siempre la articulación radio-semilunar. ${ }^{2,5,6}$

Las opciones quirúrgicas incluyen la artrodesis total de muñeca que produce una mejoría de dolor y disminución de la inflamación a costa de una pérdida de movilidad; otros procedimientos permiten conservar una movilidad relativa, fijando parte de los huesos del carpo, por ejemplo, la artrodesis de cuatro equinas y la artrodesis parcial aislada del carpo., ${ }^{2,5}$ Otra solución quirúrgica es la resección de la primera hilera del carpo o carpectomía proximal (Figuras 2 y 3), que consiste en la resección del semilunar, escafoides, piramidal, del tal manera que se constituya una nueva articulación entre el radio y la segunda fila del carpo, que se produce entre la fosa del semilunar del radio y la superficie articular del hueso grande, obteniendo una congruencia articular adecuada. ${ }^{7,8,9}$
La carpectomía proximal fue descrita originalmente por Stamm en 1994, ${ }^{9,10,11,12}$ es una técnica quirúrgica que conserva la movilidad de la muñeca para el tratamiento de procesos degenerativos como la inestabilidad de la muñeca para el tratamiento de procesos degenerativos como la inestabilidad crónica con los cambios artrósicos (SLAC), seudoartrosis crónica de escafoides con colapso del carpo (SNAC), el objetivo de este procedimiento es disminuir el dolor y mejorar el rango de movilidad y fuerza de presión al máximo posible respecto al preoperatorio. El estado de la cabeza del hueso grande y de la fosa del semilunar del radio es la clave de la indicación de esta técnica. Cualquier lesión visible en el cartílago de la cabeza del grande y del radio, que van a formar la nueva articulación radiocarpiana, contraindican esta intervención. ${ }^{13}$
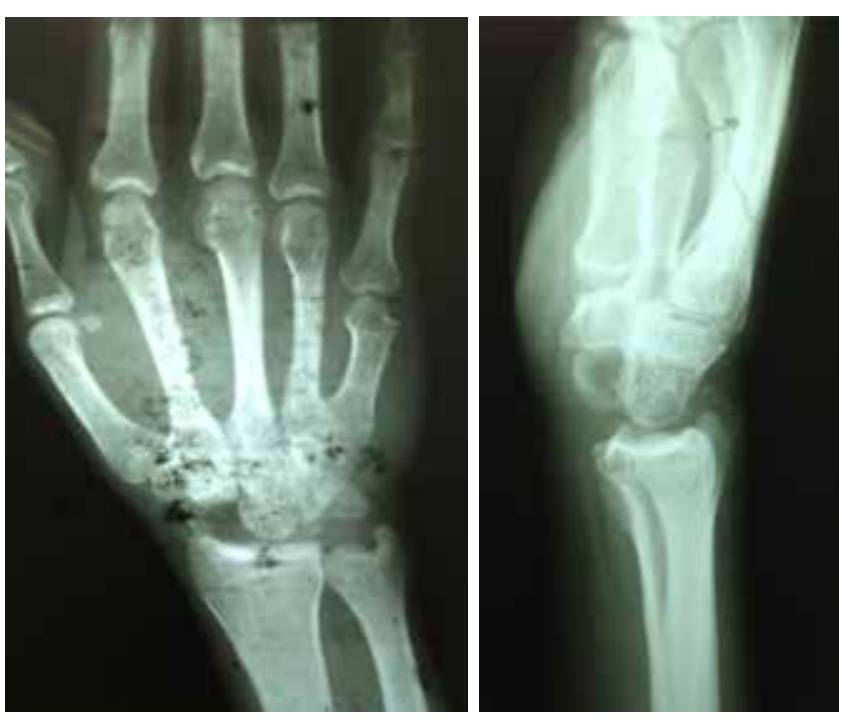

Figura 2: Carpectomía caso 1.
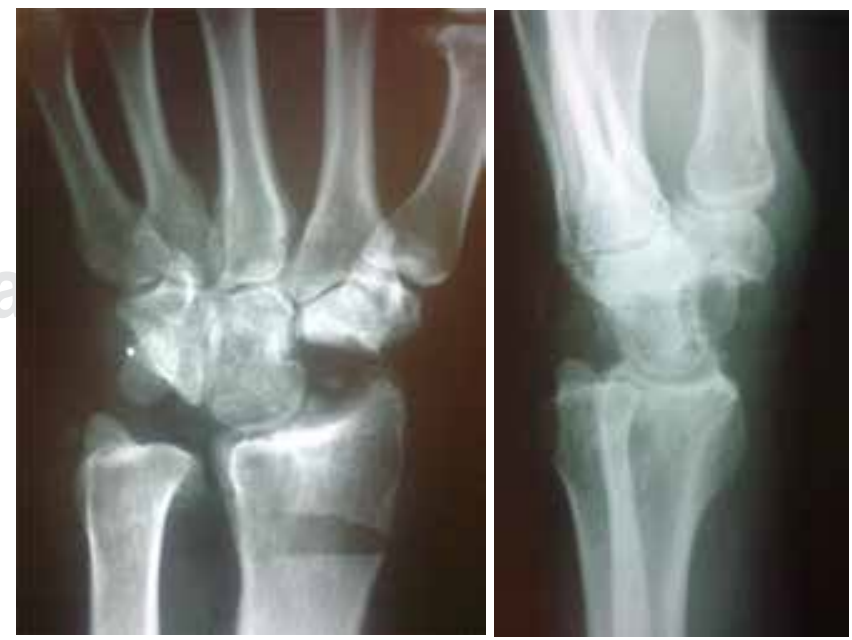

Figura 3: Carpectomía caso 2. 


\section{Material y métodos}

Se realizó un estudio observacional, descriptivo, tipo de serie de caso, ambispectivo. Tuvo como objetivo general evaluar el resultado funcional de los pacientes con artrosis de muñeca, sometidos a carpectomía proximal, mediante escalas de Mayo Wrist Score y DASH, durante el período del 1 Enero de 2007 al 31 de Agosto de 2009, en el que se efectuaron 15 carpectomías proximales, con seguimiento clínico y radiológico hasta Agosto de 2010. Se incluyeron pacientes masculinos y femeninos a quienes se les realizó carpectomía proximal por artrosis de muñeca, los cuales se detectaron mediante el registro interno del servicio. Por último, los resultados fueron evaluados mediante estadística descriptiva para las variables demográficas y las variables en estudio, producto de los cuestionarios de DASH y Mayo mediante $\chi^{2}$, Wilcoxon, Friedman, considerando estadísticamente significativo $\mathrm{p}<0.05$.

\section{Resultados}

Durante el período de Enero de 2007 a Agosto de 2009 se identificó un total de 15 pacientes con SLAC/SNAC tratados mediante resección de la fila proximal del carpo, a los cuales se dio un seguimiento hasta Agosto de 2010.

En lo referente a la edad, los 15 pacientes presentaron un promedio de 52.2 años, el paciente más joven contaba con 23 años y el paciente de mayor edad con 75, dando un rango de 52 años. Al distribuirlos por grupo de edad (en intervalos de 15 años) se observó que la mayoría (53\%) se encontraban entre 50 y 64 años, en segundo lugar (27\%) quienes tenían entre 35 y 49 años; en tercer lugar (13\%) los mayores de 65 años y sólo 7\% de los pacientes tenían 34 años o menos. Con relación a la distribución por sexo se observó que las dos terceras partes (67\%) correspondieron a pacientes del sexo masculino y el restante $33 \%$ del sexo femenino. En relación con la mano afectada se observó que la mayoría (67\%) correspondió a la mano derecha y sólo una tercera parte (33\%) a la mano izquierda. (En lo referente al tipo de artrosis, $60 \%$ fue de tipo SLAC, mientras que $40 \%$ fue de tipo SNAC.

De los nueve pacientes con tipo de artrosis SLAC, cuatro de ellos fueron grado II y cinco casos grado III. De los seis casos con tipo SNAC se encontraron tres casos grado II y tres casos grado III. De acuerdo a los resultados de la escala de Daniels en el preoperatorio y postoperatorio se aplicó la prueba no paramétrica de Wilcoxon y Friedman y se obtuvo un resultado de $\mathrm{H}=15.64 ; 1 \mathrm{gl} ; \mathrm{p}<0.05$, lo que indica que sí existe una diferencia estadísticamente significativa entre el nivel de fuerza antes y después de la resección de la fila proximal del carpo, a 95\% de confianza. Los 15 pacientes fueron evaluados en el preoperatorio y postoperatorio en relación a la extensión de la muñeca y ambos valores se refirieron en forma proporcional con respecto a la muñeca contralateral, de esta manera se pudo observar una media geométrica del porcentaje preoperatorio en relación al con- tralateral de $42 \%$, en tanto que el porcentaje postoperatorio en relación al contralateral presentó una media geométrica de $65 \%$, lo que indica un aumento de mejoría de 23 puntos porcentuales.

De acuerdo con los resultados de la escala Mayo se encontró un promedio de 26.3 con una desviación estándar de 16.8 en el preoperatorio, mientras que en el postoperatorio el promedio de la escala en los 15 pacientes fue de 66.7, valores con los cuales se calculó la t de Student pareada, dando un resultado de $p<0.05$, lo que indicó una diferencia estadísticamente significativa en la puntuación obtenida en la escala de Mayo por los pacientes al ser manejados con resección de la fila proximal del carpo.

Los valores de la escala Mayo se agruparon en las categorías: malo, satisfactorio, bueno y excelente, de este modo se observó que en el preoperatorio la totalidad de pacientes calificaron con malo, mientras que en el postoperatorio la mayoría (11 casos) se encontraron en satisfactorio; dos casos en excelente y un caso cada uno en malo y bueno respectivamente. En cuanto a la escala DASH aplicada a los pacientes en el postoperatorio se detectó $13 \%$ sin discapacidad; $20 \%$ con discapacidad leve; $47 \%$ con discapacidad moderada; así como 20\% con discapacidad severa.

\section{Discusión}

La artrosis de muñeca es un procedimiento poco frecuente; sin embargo, es sumamente incapacitante para la vida laboral y cotidiana de los pacientes, en particular a causa del dolor, que limita de manera gradual la movilidad hasta imposibilitarla. La carpectomía proximal representa una alternativa terapéutica que permite conservar la movilidad articular, con disminución del dolor y mejora de la función global de la extremidad. Los resultados satisfactorios de este estudio reportan $73 \%$ según la escala de Mayo, con una discapacidad de leve a moderada en $67 \%$ según la escala de DASH. El tiempo promedio de seguimiento en este estudio fue de 24 meses, con un máximo de 37 y un mínimo de 12. De acuerdo a la bibliografía se obtuvieron resultados similares respecto a otros autores como González y colaboradores (2009), ${ }^{13}$ quienes dieron seguimiento de 17 meses; Deise y su equipo (1996) de 24 meses, aunque existen otros seguimientos a 10 años como el de Jebson y colegas, ${ }^{14}$ pero con una muestra de 20 pacientes similar a la nuestra y concluyen que la carpectomía proximal es una buena alternativa antes de una artrodesis total de muñeca.

Respecto a los resultados obtenidos en esta serie de casos en la movilidad de la muñeca se reportan rangos mayores con una extensión promedio de $65 \%$ y una flexión de $63 \%$, a diferencia de la descrita por González y colaboradores ${ }^{13}$ la mejoría del dolor prequirúrgico y postquirúrgico fue evidente en todos los pacientes, predominante en actividades manuales de carga, en los otros autores consultados ${ }^{4,5,11,14}$ el dolor residual incapacitante estuvo presente en $10-15 \%$ de los casos. En el estudio de Tang y su equipo ${ }^{15}$ se evalúan mediante artroscopía los pacientes con dolor moderado a 
severo a 18 meses de realizada la carpectomía y concluyen que todos tenían una lesión condral severa en la fosa semilunar del radio especificando que una lesión condral incipiente es una de las causas de dolor postoperatorio.

\section{Conclusiones}

La carpectomía proximal es un procedimiento confiable que resulta en la mejoría del dolor en la mayoría los pacientes, el mantenimiento de la movilidad de la muñeca y en la mejoría en la fuerza de presión con respecto al preoperatorio. La satisfacción del paciente es regular, ya que a pesar de mejorar los rangos de la movilidad y disminuir el dolor siempre persiste sintomatología dolorosa y limitación funcional comparativamente.

La carpectomía proximal es un procedimiento quirúrgico alternativo a las artrodesis parciales o totales de la muñeca para aquellos pacientes que desean preservar la movilidad radio carpiana con disminución de dolor y que no requieran de alta demanda física para sus actividades cotidianas y laborales.

\section{Bibliografía}

1. Calandruccio JH. Proximal row carpectomy. J Hand Surg Am. 2001; 2: 112-22.

2. Cohen MS, Kozin SH. Degenerative arthritis of the wrist: proximal row carpectomy versus scaphoid excision and four corner arthrodesis. J Hand Surg Am. 2001; 26: 94-104.
3. Watson HK, Ballet FL. The SLAC writs: scapholunate collapse pattern of degenerative arthritis. J Hand Surg. 1984; 9A: 348-65.

4. Hogan CJ, Mckay PL, Degnan GG. Changes in radiocarpal loading characteristics after a proximal row carpectomy. J Hand Surg Am. 2004; 29: 1109-13.

5. Di Donna ML, Kierfhaber Tr, Sern PJ. Proximal row carpectomy: a study with a minimum of ten years of follow-up. J Bone Joint Surg Am. 2004; 86: 2359-65.

6. Ferlic DC, Clayton ML, Mills MF. Proximal row carpectomy: review of rheumatoid and non rhematoid wrists. J Hand Surg Am. 1991; 16: 420-24.

7. Lucchetti R, Soragni O, Fairplay T. Proximal row carpectomy throught a palmar approach. J Hand Surg Br. 1998; 23: 406-9.

8. Stern PJ, Agabegi SS, Kiefhaberb TR, Diddona ML. Proximal row carpectomy: surgical technique. J Bone J Surg. 2005; 87: 166-74.

9. Imbriglia J, Broudy A, Hegberg WC, Mckernan D. Proximal row carpectomy: clinical evaluation. J Han Surg. 1990; 1292-300.

10. Oishi S, Muzaffar AR, Carter P. Treatment of Kienbock's disease with capitohamate arthrodesis: pain relief with minimal morbidity. Plast Reconstr Surg. 2002; 109: 1292-300.

11. Rios LA, Villanueva MM, Fahandezh-Saddi D. Is proximal row carpectomy a good operation for Kienböck disease? Patalogia del Aparato Locomotor. 2006; 4(Supl. 1): 58-63.

12. Escribano RJ. Resección de la primera hilera proximal del carpo: indicaciones y resultados. Revista Española de Cirugía Osteoarticular. 2008; 234(44): 231-65.

13. González CC, Carlos GB, Giraldo MM, Ramirez MJ, Felipe HT. Resultados funcionales de carpectomía proximal en estadios avanzados en paciente con enfermedad de Kienbock. Rev Col Or Tra. 2009; 23(1): 21-6.

14. Jebson PJ, Hayes EP, Engber WD. Proximal row carpectomy: a minimum of 10 year follow-up study. J Hand Surg Am. 2003; 28: 56169.

15. Tang P, Imbriglia J. Osteochondral resurfacing for capitate chondrosis in proximal row carpectomy. J Hand Surg. 2007; 32A: 1334-42. 